\title{
Erratum
}

\section{Erratum: Li et al., "Nerve Injury-Induced Neuronal PAP-I Maintains Neuropathic Pain by Activating Spinal Microglia"}

In the article "Nerve Injury-Induced Neuronal PAP-I Maintains Neuropathic Pain by Activating Spinal Microglia" by Jiayin Li, Haixiang Shi, Hui Liu, Fei Dong, Zhiping Liu, Yingjin Lu, Luonan Chen, Lan Bao, and Xu Zhang, which appeared on pages 297-310 of the January 8, 2020 issue, author affiliation 2 was incorrect. The affiliation should appear as, "State Key Laboratory of Cell Biology, CAS Center for Excellence in Molecular Cell Biology, Institute of Biochemistry and Cell Biology, University of Chinese Academy of Sciences, Chinese Academy of Sciences, Shanghai 200031, China".

The online version has been corrected.

DOI: 10.1523/JNEUROSCI.0064-20.2020 